\title{
Defining the risk in health insurance
}

To the editor - Although one would not wish to underestimate the potential for further unfair discrimination against people with long-term medical disorders your editorial (Genes and Discrimination. Nature Medicine 1, 385) appears, for perhaps understandable reasons, to be influenced heavily by North American experience.

Whilst it is certainly true that insurance companies routinely deny full health insurance coverage to people with pre-existing conditions, especially when such people seek travel insurance, the evidence that insurance companies are predisposed to discriminate against the sick "whenever possible" is not fully borne out by experience in the United Kingdom.
Our experience is that the main problem is not a cynical desire to deny people insurance regardless of risk, rather a profound ignorance of what risk is involved. The insurance industry needs to understand, for example, the difference between being a carrier of a recessive disorder, such as cystic fibrosis, having the disease itself or carrying a gene for a lateonset disorder such as Huntington's chorea. Before allowing ourselves to be carried away by the slippery slope argument, we should ask ourselves whether a concerted effort to engage in dialogue with the insurance industry might not bring dividends. After all the logical outcome of the discovery of more and more genes is that we shall all become suspect in the eyes of potential insurers and no one will ever be insured again; a somewhat unlikely outcome.

Insurers, like the general public (to which they belong), need to be educated. We all have a role to play in that and we should begin by getting the facts right. With great respect, people who carry the gene for cystic fibrosis are not, as stated in your editorial, predisposed to cystic fibrosis. Carriers are symptom-free. It is the children of parents who are both carriers who run the risk of having cystic fibrosis.

\section{PETER KENT}

Family and Adult Support Services

Cystic Fibrosis Trust

Alexandra House

5 Blyth Road

Bromley, Kent BR1 3RS, UK

\section{Bromocriptine in the treatment of alcoholics}

To the editor - In the April issue of Nature Medicine, Lawford et al. (Bromocriptine in the treatment of alcoholics with the D2 dopamine receptor $\mathrm{A} 1$ allele. Nature Medicine 1, 337-341) emphasize differences observed in subjective responses to bromocriptine in certain alcoholic patient subgroups and at certain times. However, their data do not support a conclusion that the A1 allele (which is due to a restriction site polymorphism well outside the $D R D 2$ coding sequence) predicts relapse after bromocriptine treatment. Nor do their data indicate that bromocriptine is efficacious in the treatment of alcoholism. Over the six-week duration of the study, 14 out of $31(0.45)$ placebo-treated alcoholics relapsed and $17 / 52(0.33)$ bromocriptine-treated alcoholics relapsed $\left(\chi^{2}=0.8, n s\right)$. Further, craving appears to have diminished to about the same extent in the placebo and bromocriptine-treated groups.

There are also problems with the one positive finding: that craving and anxiety scores following bromocriptine treatment are affected by the $\mathrm{A} 1$ allele. It is interesting, for a paper concerning relationships between genotypes and phenotypes, that genotypes are not actually presented, nor can they be deduced from the data supplied. Comparisons may have been 'preplanned' but there are a lot of them, they are performed and presented piecemeal, and there is apparently no correlation for multiple testing.
The 'significant' A1 allele effects on anxiety and craving were also found at different times (see table).

Focusing on craving, a variable that may be relevant to relapse, there are no effects at three weeks regardless of treatment or A1 allele status. Over the six-week duration of the study, group compositions changed. By the time a significant difference in craving emerges between $\mathrm{A} 1$ - and $\mathrm{A} 1+$ alcoholics treated with bromocriptine, the difference can easily be attributed to subject attrition. Group differences in craving can arise because of the subtraction of variable numbers of subjects who were higher or lower in craving. For this reason, it is more meaningful to assess the effect of bromocriptine on craving, anxiety and depression at three weeks, when the drug has had a chance to work, but before six weeks, when group compositions may have been distorted by attrition.

After bromocriptine treatment and across the six-week duration of the study, relapse rates were 5 out of $19(0.26)$ for the bromocriptine $\mathrm{A} 1+$ group and 12 out of 33 (0.36) for the bromocriptine A1- group (not significantly different). There was also no significant effect on relapse for A1 allele status by treatment and with all four groups: bromocriptine $\mathrm{A} 1+$, bromocriptine A1-, placebo $\mathrm{A} 1+$ and placebo $\mathrm{A1}-$ (d.f. $=3, \chi^{2}=0.047, P=0.83$ ).

Thus their conclusion should have been that no direct evidence is provided for an effect of bromocriptine on relapse; exploratory analyses indicate that DRD2 A1 allele/bromocriptine interactions on anxiety and craving may be observable at certain (albeit different) times; and if bromocriptine treatment works, the DRD2 A1 allele does not predict which alcoholics should receive it.

\section{DAVID GOLDMAN}

Laboratory of Neurogenetics,

National Institute on Alcohol Abuse and Alcoholism

Rockville, Maryland 20852, USA

Noble et al. reply - Evidence in the literature indicates that bromocriptine does not manifest its effect on craving in alcoholics until after three weeks of treatment. A similar lag period is also found in the action of other psychoactive medications including antidepressants and neuroleptics. Thus, on an $a$ priori basis, we studied in a doubleblind bromocriptine (BRO)/placebo (PLA) trial, two treatment periods separately: 0-3 weeks and 3-6 weeks. The results showed that in the $0-3$ week period, no differences in decreased craving or attrition were found among the four groups of alcoholics (BRO A1+, BRO A1-, PLA A1+, PLA A1-). On the other hand, when the $3-6$ week period was analysed (a time when BRO began to exert its effect), significant differences were found in these measures. Specifically, the alcoholics receiving BRO and carrying 